\title{
Período de incubação da ferrugem do cafeeiro*
}

\author{
Waldenilza Monteiro Vital Alfonsi ${ }^{1} \oplus$, Priscila Pereira Coltri ${ }^{2} \oplus$, Jurandir Zullo Júnior ${ }^{2} \oplus$, \\ Flávia Rodrigues Alves Patrício ${ }^{3}$, Eduardo Lauriano Alfonsi ${ }^{2}$ E
}

${ }^{1}$ Universidade Estadual de Campinas-UNICAMP, Faculdade de Engenharia Agrícola-FEAGRI, Av. Cândido Rondon, no. 501, Cidade Universitária, CEP 13083-875 Campinas, SP, Brazil. ${ }^{2}$ Universidade Estadual de Campinas-UNICAMP, Centro de Pesquisas Meteorológicas e Climáticas Aplicadas a Agricultura-CEPAGRI, Avenida André Tosello, 209, Cidade Universitária “Zeferino Vaz", CEP 13083-886 Campinas, SP, Brazil. ${ }^{3}$ Instituto Biológico, Alameda dos Vidoeiros, $n^{\circ}$. 1097, Sítios de Recreio Gramado, CEP 13101-680 Campinas, SP, Brazil. *Parte do projeto de doutorado junto à Faculdade de Engenharia Agrícola-Unicamp

Autor para correspondência: Waldenilza Monteiro Vital Alfonsi (walmvital@gmail.com)

Data de chegada: 29/10/2017. Aceito para publicação em: 04/02/2019.

$10.1590 / 0100-5405 / 187216$

\section{RESUMO}

Alfonsi, W.M.V.; Coltri, P.P.; Zullo Júnior, J.; Patrício, F.R.A.; Alfonsi, E.L. Período de incubação da ferrugem do cafeeiro. Summa Phytopathologica, v.45, n.2, p.134-140, 2019.

O café é uma das culturas com grande expressão mundial e, dentre as principais doenças, a ferrugem do cafeeiro (Hemileia vastatrix Berk. $\&$ Br.), é considerada a mais importante. Uma das formas de avaliar a severidade da ferrugem é por meio de equações que estimam o período de incubação (PI) do fungo. O presente estudo teve o objetivo de revisar os principais trabalhos sobre a ferrugem do cafeeiro e o período de incubação, de maneira a elucidar as principais equações desenvolvidas para esse fim.
Na literatura, encontrou-se dez equações que estimam os valores do PI, para diferentes condições. A maioria dos estudos brasileiros utilizam a equação de Moraes et al. (31), desenvolvidas para cultivo a pleno sol e sombreado, em condição de macroclima. O estudo do PI é utilizado em diferentes níveis hierárquicos e em diferentes cenários climáticos sendo importante para tomadas de decisão e políticas públicas, auxiliando agricultores no planejamento e gerenciamento da área.

Palavras-chave: Hemileia vastatrix, doença café, equações

\section{ABSTRACT}

Alfonsi, W.M.V.; Coltri, P.P.; Zullo Júnior, J.; Patrício, F.R.A.; Alfonsi, E.L. Incubation period of coffee leaf rust. Summa Phytopathologica, v.45, n.2, p.134-140, 2019.

Coffee is one of the world's most famous crops and, among its major diseases, coffee leaf rust (Hemileia vastatrix Berk. \& Br.) is considered most important. One of the ways to evaluate rust severity is by means of equations that estimate the incubation period (IP) of the fungus. The present study aimed to review the main studies on coffee leaf rust and its incubation period, in order to elucidate the main equations developed for this purpose. In the literature, there are at least ten equations that estimate IP values for different conditions. Most of the Brazilian studies have used the equation of Moraes et al. (31), developed for full sun and shade cultivation, under macroclimate condition. The study of IP is used at different hierarchical levels and in different climatic scenarios and is important for decision making and public policy, assisting farmers in the planning and management of the area.

Keywords: Hemileia vastatrix, coffee disease, equations

O café é o produto tropical que mais se negocia no mundo e, mesmo ainda sendo considerado uma "commodity", vem ganhando status de especialidade ("speciality") no mercado internacional, em razão das exigências cada vez maiores dos consumidores. Em todo o mundo, são tomadas mais de 500 bilhões de xícaras de café por dia (12). Produzido em mais de 70 países (7), sustenta 120 milhões de pessoas, sendo a maioria de pequenos cafeicultores dos países em desenvolvimento (20).

No Brasil, a cafeicultura é um dos setores da economia que contribui decisivamente na formação do Produto Interno Bruto (PIB) nacional, gerando empregos diretos e indiretos e colaborando com o aumento da renda do trabalhador rural. A safra de 2016 foi responsável por 35,5\% do mercado internacional, totalizando 56,8 milhões de sacas produzidas (21). O país é o maior produtor mundial do grão, seguido do Vietnã e da Colômbia, com 25,5 e 14,6 milhões de sacas, respectivamente (21).

Dentre as principais doenças do café, a ferrugem do cafeeiro (Hemileia vastatrix Berk. \& Br.), é considerada a mais importante, uma vez que, em regiões onde as condições climáticas são favoráveis, os prejuízos na produção podem chegar a mais de $50 \%(46,37)$, sendo muitas vezes devastadores no cafezal.

A velocidade com que o patógeno se reproduz, está diretamente associada com a severidade da doença (31). O período de incubação (PI), que é compreendido pelo tempo entre a germinação e a penetração do fungo nos tecidos vegetais até o aparecimento dos primeiros sintomas $(42,31,8,46,27)$, pode ajudar a explicar este fato.

O período de incubação é influenciado por fatores ambientais, principalmente pela temperatura $(40,33,41,32,3,11,39)$. Regiões com temperaturas mais elevadas apresentam menores valores do período de incubação, sugerindo que o ciclo do patógeno pode diminuir, indicando maior probabilidade na severidade da doença. Por outro lado, regiões com temperaturas amenas tendem a apresentar valores do período de incubação maiores, indicando menor probabilidade de incidência da ferrugem do cafeeiro (39).

A maioria dos trabalhos que analisam PI, utilizam a equação desenvolvida por Moraes et al. (31), mas existem na literatura, pelo 
menos outras nove equações que também estimam PI, e podem ser utilizadas em diferentes condições, como por exemplo em condições de cultivo sombreado, pleno sol, com a utilização de dados de macroclima e de microclima. A análise do período de incubação da ferrugem utilizando modelos de regressão data de 1960, quando as primeiras equações surgiram.

Esse trabalho tem por objetivo revisar e discutir os principais trabalhos sobre a ferrugem do cafeeiro e o período de incubação, de maneira a elucidar as principais equações desenvolvidas para esse fim e como essas podem ser utilizadas em trabalhos da ferrugem do cafeeiro.

\section{Histórico da Ferrugem do Cafeeiro}

A ferrugem foi encontrada pela primeira vez no mundo em 1861, em cafeeiros silvestres, próximo ao Lago Victória, no Quênia, África Oriental $(9,35)$. O fungo Hemileia vastatrix Berk. \& Br, foi descrito pela primeira vez por Berkeley $(9,35)$. Em 1867, apareceu no Ceilão, hoje Sry-Lanka, e, dois anos após, em 1869, a doença já demonstrava gravidade $(9,14,24,35)$. Sua devastação foi tamanha que o país deixou o cultivo do cafeeiro e passou a cultivar o chá (27).

A ferrugem do cafeeiro provoca queda precoce das folhas, seca de ramos, que não produzirão frutos no ano seguinte. Os primeiros sintomas da doença são manchas cloróticas com 1 a 3 mm de diâmetro $(11,13,48)$, sendo que, posteriormente, formam-se pequenas manchas circulares de coloração amarelo-alaranjadas, na face inferior da folha, podendo atingir até $2 \mathrm{~cm}$ de diâmetro (48) e, sobre a mancha, forma-se uma massa pulverulenta de uredósporos. Na face superior das folhas, é possível observar manchas cloróticas amareladas, que necrosam no estágio mais avançado $(13,48)$. Quando a infestação é acentuada, a doença causa desfolha, podendo atrasar o desenvolvimento e comprometer a produção (48).

Levantamentos realizados, narram que a ferrugem do cafeeiro foi constatada em diversos locais da Ásia e Índia entre 1869 e 1966, sendo eles: Índia (em 1869), em Sumatra (em 1876), nas Ilhas Fiji (em 1878), em Java (em 1879), no Vietnã (em 1888), nas Filipinas, em Bornéu (em 1890) e na Nova Caledônia (em 1910), nas Ilhas Maurício (em 1886), Madagascar (em 1886), Tanganica (em 1894) e Rodésia (em 1916). Em 1918, foi constada na bacia do Congo. Mais tarde, em meados de 1951-1952, foi detectada na República de Camarões, Daomé, Togo, Costa do Marfim, Libéria, Guiné, Nigéria e Angola (1966) (35).

Nas Américas, os primeiros relatos de ferrugem foram na Ilha de Porto Rico, em 1902 (14). Segundo observações (46, 48, 24, 14), a ferrugem do cafeeiro é uma doença bastante antiga nos cafezais brasileiros, tendo sido constatada no Brasil por Medeiros (26), em janeiro de 1970, no Sul da Bahia (14, 24, 46, 48), quando examinava plantas de café junto às plantações de cacau (24). Quatro meses após a primeira constatação, no mês de maio $(46,48)$, a doença foi encontrada em quase todos os estados brasileiros onde se cultivava o café $(5,14$, $24,46)$ demonstrando o rápido potencial de disseminação do fungo.

Depois do Brasil, a ferrugem continuou infectando outros países. Em 1973, foi constada no Paraguai e nas regiões das Missões na Argentina $(35,19,1)$; em 1976, na Nicarágua; em 1977, no Peru; em 1979, em El Salvador; em 1980, na Guatemala (38); em 1981, no México; em 1983, na Costa Rica e, em 1984, na Colômbia e Venezuela (46).

Segundo os autores (24 e 14), é possível que a ferrugem do café tenha vindo do continente africano, já que a primeira observação nas Américas ocorreu no Brasil. Existem duas hipóteses quanto à chegada da ferrugem: a primeira, é que ela veio através de esporos sobre as mudas de cacau trazidas da África; e, a segunda, é que ela chegou através dos esporos transportados por correntes aéreas vindas também da África.

Atualmente, já foram descritas mais de 50 raças fisiológicas de Hemileia vastatrix, sendo quinze delas já descritas no Brasil, no gênero Coffea arabica $(15,45,49)$. Devido à alta variabilidade do patógeno e à grande expansão das áreas de cultivo, é possível que existam outras mais (45). Embora já tenham sido realizados inúmeros estudos sobre a ferrugem do cafeeiro, ainda existem muitas dúvidas sobre esse fungo.

O fungo Hemileia vastatrix ataca todas as variedades de café do gênero Coffea, sendo observadas diferenças de patogenicidade. A maioria das cultivares comerciais da espécie Coffea arábica L. são suscetíveis à ferrugem (14). Hoje, a ferrugem do cafeeiro está presente em todas as regiões produtoras de café do mundo, sendo considerada a principal doença da cultura. Onde as condições climáticas são favoráveis à doença, os prejuízos no Brasil atingem 35\% (14, 37). Em condições de estiagem prolongada, nos períodos de maior severidade da doença, as perdas podem chegar a mais de $50 \%$ da produção, dependendo da cultivar, da nutrição das plantas e do clima predominante da região de cultivo $(14,46,47,48)$. A severidade da doença está associada a velocidade com que o patógeno se reproduz (31).

\section{Período de Incubação da Ferrugem do Cafeeiro - PI}

Devido a grande preocupação com o destino da cafeicultura mundial em relação ao ataque da ferrugem do cafeeiro, diversos estudos foram conduzidos com o intuito de conhecer e/ou controlar essa doença que devastou com grande rapidez as lavouras cafeeiras de vários países. Desde 1861 a ferrugem do cafeeiro tem sido estudada, entretanto, a doença ainda merece muita atenção, pois em regiões com condições climáticas favoráveis, hospedeiros suscetíveis e sem controle, pode ser devastadora, como já ocorrido no Brasil em 1970, recentemente na Colômbia e América Central.

Desde então pesquisadores de diferentes áreas, formaram grupos de estudo da ferrugem do café em todo o mundo. Com o intuito de tentar resolver e/ou conhecer o comportamento da ferrugem em condições de campo, pesquisadores desenvolveram equações de regressão que estimam o período de incubação (PI) da ferrugem do cafeeiro. Essas equações utilizam dados de temperatura máxima, temperatura mínima $(40,31,22)$ e temperatura média (34).

O PI é o período compreendido pelo tempo entre a germinação e a penetração do fungo nos tecidos vegetais até o aparecimento dos primeiros sintomas $(8,30,31,42,46)$. Quanto menor o PI maior poderá ser a intensidade da epidemia (17).

O PI da ferrugem do cafeeiro foi estudado em países como Angola, África do Sul, Brasil, Ceilão, Colômbia, Índia, Quênia e Uganda. Os primeiros dados sobre do período de incubação da ferrugem do cafeeiro foram divulgados em 1882 (44) no Ceilão. Posteriormente, em 1930, na Índia, o PI da ferrugem foi estudado em condições de laboratório, com cafeeiros da espécie Coffea arábica (25). Já em condições de campo, os primeiros estudos foram desenvolvidos em 1961 (40) no Quênia. Nesse estudo, o autor desenvolveu uma equação para avaliar o potencial de infecção da ferrugem, sendo que a temperatura foi a variável que mais afetou o período de incubação. Os valores do período de incubação (40), no Quênia, variavam de 27 a 47 dias. A equação 1 (40) para estimativa do número de dias para início da esporulação em $50 \%$ das lesões em função da temperatura média das máximas e mínimas, em graus Fahrenheit (Tabela 1).

Os valores dos PIs encontrados no Quênia são maiores (40) que os observados no Ceilão (44) e na Índia (25). O autor (40) atribui esse fato, às diferentes condições térmicas presentes nos locais estudados. 
Tabela 1. Equações do período de incubação, autores e especificações.

\begin{tabular}{|c|c|c|c|}
\hline $\mathbf{N}^{\mathbf{0}}$ & Autor & Equação & Especificações \\
\hline & & $Y=90,61-0,408 \times 1-0,440 \times 2$ & \\
\hline 1 & Rayner (1961) & $\begin{array}{l}\text { Onde: } \mathbf{Y}=\text { número de dias para início da esporulação em } 50 \% \text { das lesões; } \\
\mathbf{X 1}=\text { temperatura média das máximas, em graus Fahrenheit; e } \mathbf{X} \mathbf{2}= \\
\text { temperatura média das mínimas, em graus Fahrenheit. }\end{array}$ & $\begin{array}{l}\text { Desenvolvida nas condições do Quênia, África. } \\
\text { Geralmente no Brasil, tende a aumentar a severidade }\end{array}$ \\
\hline 2 & Ortolani et al. (1971) & $\begin{array}{l}\mathrm{Y}=\mathbf{6 3 , 4 7}-\mathbf{1 , 5 2 6} \mathrm{X} \\
\text { Onde: } \mathrm{Y}=\text { estimativa do período de incubação, em dias; } \mathrm{X} 1=\text { temperatura } \\
\text { média máxima, em }{ }^{\circ} \mathrm{C} ; \text { e } \mathrm{X} 2=\text { temperatura média mínima, em }{ }^{\circ} \mathrm{C} \text {. }\end{array}$ & $\begin{array}{l}\text { Desenvolvida nas condições Brasileiras, para os estados } \\
\text { de São Paulo e Minas Gerais. Foi desenvolvida a partir } \\
\text { da equação de Rayner (1961) }\end{array}$ \\
\hline 3 & Moraes et al. (1976) & $\begin{array}{l}\mathrm{Y}=\mathbf{1 0 3 , 0 1}-\mathbf{0 , 9 8} \times \mathbf{1}-\mathbf{2 , 1 0} \times \mathbf{2} \\
\text { Onde: } \mathrm{Y}=\text { estimativa do período de incubação, em dias; X1 = temperatura } \\
\text { média máxima, em }{ }^{\circ} \mathrm{C} ; \text { e } \mathrm{X} 2=\text { temperatura média mínima, em }{ }^{\circ} \mathrm{C} \text {. }\end{array}$ & $\begin{array}{l}\text { Equação desenvolvida nas condições brasileiras, para } \\
\text { cultivo do café a pleno sol+sombreado, em condições } \\
\text { de macroclima }\end{array}$ \\
\hline 4 & Moraes et al. (1976) & $\begin{array}{l}\mathrm{Y}=\mathbf{9 3 , 2 7}-\mathbf{0 , 9 9} \mathrm{X} 1-\mathbf{1 , 5 1} \mathbf{X} 2 \\
\text { Onde: } \mathrm{Y}=\text { estimativa do período de incubação, em dias; X1 = temperatura } \\
\text { média máxima, em }{ }^{\circ} \mathrm{C} ; \text { e X2 = temperatura média mínima, em }{ }^{\circ} \mathrm{C} \text {. }\end{array}$ & $\begin{array}{l}\text { Equação desenvolvida nas condições brasileiras, } \\
\text { para Cultivo do café a pleno sol, em condições de } \\
\text { macroclima }\end{array}$ \\
\hline 5 & Moraes et al. (1976) & $\begin{array}{l}\mathrm{Y}=\mathbf{1 1 3 , 9 2}-\mathbf{1 , 0 2} \times 1-\mathbf{2 , 6 9} \times \mathbf{2} \\
\text { Onde: } \mathrm{Y}=\text { estimativa do período de incubação, em dias; } \mathrm{X} 1=\text { temperatura } \\
\text { média máxima, em }{ }^{\circ} \mathrm{C} \text {; e } \mathrm{X} 2=\text { temperatura média mínima, em }{ }^{\circ} \mathrm{C} \text {. }\end{array}$ & $\begin{array}{l}\text { Equação desenvolvida nas condições brasileiras, } \\
\text { para cultivo do café a sombreado, em condições de } \\
\text { macroclima }\end{array}$ \\
\hline 6 & Moraes et al. (1976) & $\begin{array}{l}\mathrm{Y}=\mathbf{8 7 , 6 8}-\mathbf{0 , 4 0} \mathrm{X} \mathbf{1}-\mathbf{2 , 1 6} \mathrm{X} \mathbf{2} \\
\text { Onde: } \mathrm{Y}=\text { estimativa do período de incubação, em dias; } \mathrm{X} 1=\text { temperatura } \\
\text { média máxima, em }{ }^{\circ} \mathrm{C} \text {; e } \mathrm{X} 2=\text { temperatura média mínima, em }{ }^{\circ} \mathrm{C} \text {. }\end{array}$ & $\begin{array}{l}\text { Equação desenvolvida para condições brasileiras para } \\
\text { cultivo de café pleno sol+sombreado em condições de } \\
\text { microclima }\end{array}$ \\
\hline 7 & Moraes et al. (1976) & $\begin{array}{l}\mathrm{Y}=\mathbf{7 4 , 7 2}-\mathbf{0 , 1 1} \mathrm{X} 1-\mathbf{1 , 9 3} \mathrm{X} 2 \\
\text { Onde: } \mathrm{Y}=\text { estimativa do período de incubação, em dias; } \mathrm{X} 1=\text { temperatura } \\
\text { média máxima, em }{ }^{\circ} \mathrm{C} ; \text { e } \mathrm{X} 2=\text { temperatura média mínima, em }{ }^{\circ} \mathrm{C} \text {. }\end{array}$ & $\begin{array}{l}\text { Equação desenvolvida para condições brasileiras } \\
\text { em cafezais cultivados a pleno sol, em condições de } \\
\text { microclima }\end{array}$ \\
\hline 8 & Moraes et al. (1976) & $\begin{array}{l}\mathrm{Y}=\mathbf{1 3 8}, \mathbf{4 0}-\mathbf{2 , 3 6} \mathrm{X} \mathbf{1}-\mathbf{1 , 7 8} \times \mathbf{2} \\
\text { Onde: } \mathrm{Y}=\text { estimativa do período de incubação, em dias; } \mathrm{X} 1=\text { temperatura } \\
\text { média máxima, em }{ }^{\circ} \mathrm{C} ; \text { e } \mathrm{X} 2=\text { temperatura média mínima, em }{ }^{\circ} \mathrm{C} \text {. }\end{array}$ & $\begin{array}{l}\text { Equação desenvolvida para condições brasileiras } \\
\text { em cafezais de cultivo sombreado, em condições de } \\
\text { microclima }\end{array}$ \\
\hline 9 & $\begin{array}{l}\text { Kushalappa e Martins } \\
\text { (1980) }\end{array}$ & $\begin{array}{l}\mathrm{Y}=\mathbf{1 0 0 , 8 9 4}+\mathbf{0 , 0 8 8} \mathrm{X} \mathbf{1}-\mathbf{4 , 0 8 4} \mathrm{X} \mathbf{2} \\
\text { Onde: } \mathrm{Y}=\text { estimativa do período de incubação, em dias; } \mathrm{X} 1=\text { temperatura } \\
\text { média máxima, em }{ }^{\circ} \mathrm{C} ; \text { e } \mathrm{X} 2=\text { temperatura média mínima, em }{ }^{\circ} \mathrm{C} \text {. }\end{array}$ & $\begin{array}{l}\text { Equação desenvolvida nas condições da cidade de } \\
\text { Viçosa - MG, em condições de macroclima }\end{array}$ \\
\hline 10 & $\begin{array}{l}\text { Kushalappa e Martins } \\
\text { (1980) }\end{array}$ & $\begin{array}{l}\mathrm{Y}=\mathbf{5 3 , 1 2}+\mathbf{1 , 7 7 9} \mathrm{X} \mathbf{1}-\mathbf{3 , 9 8 7} \mathrm{X} \mathbf{2} \\
\text { Onde: } \mathrm{Y}=\text { estimativa do período de incubação, em dias; } \mathrm{X} 1=\text { temperatura } \\
\text { média máxima, em }{ }^{\circ} \mathrm{C} ; \text { e } \mathrm{X} 2=\text { temperatura média mínima, em }{ }^{\circ} \mathrm{C} \text {. }\end{array}$ & $\begin{array}{l}\text { Equação desenvolvida nas condições da cidade de } \\
\text { Viçosa -MG, em condições de microclima }\end{array}$ \\
\hline
\end{tabular}

Baseado na equação 1 (40), outro estudo (43), propõe uma escala para avaliar o potencial de infecção da ferrugem do cafeeiro, sugerindo 3 graus de severidade da ferrugem, em função da duração do PI: risco elevado de ataque severo: PI inferior a 20 dias; risco médio de ataque severo: PI entre 20 e 30 dias; e baixa probabilidade de risco de ataque severo: PI superior a 30 dias.

No entanto, essa equação, desenvolvida no Quênia, sendo muito restrita para as condições brasileiras, e consequentemente, as classes de severidade da ferrugem do cafeeiro (43). Essas classes também são muito restritivas para estudos de PI no Brasil.

Estudos mais recentes, regionalizados, no Brasil, utilizando diferentes equações de PI para os estados de São Paulo e Minas Gerais (1), também verificaram que a equação 1 (40) é muito restritiva para as condições brasileiras, e, assim, quando utilizam a classificação (43), a severidade é sempre elevada. Diante desse contexto foi proposta uma nova escala de grau de severidade, segundo a duração do PI (1). Essa nova escala levou em consideração as equações de PI 1, 2, 3, 4, 5, 6, $7,8,9$ e $10(40,34,31,22)$, e apresenta seis novas classes, conforme Tabela 2 (1).

Em expedição oficial, realizada em Angola, África do Sul, Quênia, Tanzânia e Uganda (34), demostram a variabilidade dos valores estimados de PI da ferrugem do cafeeiro, nesses países. Em Angola os valores de PI variam de 29 a 32 dias de outubro a março, e de 33 a 36 dias para os meses mais secos. Os índices mais críticos do PI foram de janeiro a maio, onde os valores de PI foram inferiores a 28 dias e de outubro a dezembro de 29 dias, coincidindo com o período chuvoso. Em Amboim o PI foi de 32 dias, Carmona de 29 dias, Ganda de 32 dias, Nova Lisboa de 35 dias e Salazar de 29 dias. O PI médio de Angola foi de 31 dias. Baseado nas classes de severidade em função do PI (43), em Amboim, Ganda e Nova Lisboa o risco de probabilidade de ataque 
Tabela 2. Potencial de infecção da ferrugem do cafeeiro em relação à duração do período de incubação (1).

\section{Período de Incubação (dias) Potencial de Infecção}

PI menor ou igual 19 dias

PI entre 20 - 29 dias

PI entre 30 - 39 dias

PI entre 40 - 49 dias

PI entre 50 - 59 dias

PI acima de 60 dias
Muito Alto

Alto

Médio

Baixo

Muito Baixo

Nulo severo da ferrugem é considerado baixo, PI superiores a 30 dias e para as regiões de Carmona e Salazar, o risco de ataque é médio.

O PI estimado para as regiões da África do Sul variam de 28 a 40 dias, sendo o PI médio de 33 dias. Em Nelspruit, o PI médio é 29,3 dias (outubro- março), onde o potencial de infecção da ferrugem apresenta risco médio de ataque severo (34).

No Quênia, regiões de Nakuru, Kericho, Kisii e Ruiru, os valores médios de PI foram maiores de 30 dias (37, 35, 30 e 35 dias, respectivamente). Em Rift Valley, de janeiro a março os valores de PI são de 31 dias e para os demais meses de 32 e 35 dias. Em Kisumu, o PI variou de 27 a 30 dias. Em Uganda, região de Kawanda, os valores médios do PI foram de 31 dias. Considerando o potencial de infecção da ferrugem, (43), onde o autor sugere que PIs superiores a 30 dias, a probabilidade de risco de ataque severo é baixa.

Nas regiões de Nakuru e Kericho, a ferrugem não encontra condições favoráveis ao desenvolvimento. O total de chuva anual é de $1033 \mathrm{~mm}$ para Nakuru e de $1601 \mathrm{~mm}$ para Kericho e, a distribuição uniforme nos períodos de abril-novembro e fevereiro-dezembro, respectivamente (34).

Os primeiros estudos do PI em condições de campo no Brasil, foram realizados em 1971 (34), utilizando a equação 1 (40), os autores calcularam os valores do PI da ferrugem do cafeeiro para as condições brasileiras, conforme. Os autores selecionaram municípios tradicionais no cultivo do café para a época. Esses municípios fazem parte dos estados de São Paulo e Paraná. Os valores médios do período de incubação da ferrugem observados foram de 28 dias para o município de Araçatuba, PI de 29 dias para Pindorama e Presidente Prudente, PI de 30 dias para Maringá e Ribeirão Preto, PI de 31 dias para Campinas e Londrina, PI de 32 dias para Franca e PI de 33 dias para Monte Alegre do Sul. O efeito regional da altitude pode ser verificado nos dados de Franca e Monte Alegre do Sul e Campinas com 1036, 777 e $663 \mathrm{~m}$ de altitude, respectivamente. Para esses municípios, os menores valores de PI, inferiores a 30 dias, ocorrem no período chuvoso, pois incremento na temperatura tendem a diminuir os valores de PI. Já para Ribeirão Preto, Pindorama e Araçatuba, onde a altitude é menor e a temperatura é maior, os valores de PI encontrados oscilaram ente 24 e 28 dias, demostrando a relação das variáveis climáticas com o PI (34).

Para o estado do Paraná, município de Londrina e Maringá, apresentam acentuada amplitude térmica. A partir de outubro-novembro apresenta aumento da doença, atingindo índices máximos em fevereiro. Durante o período do inverno, pode ocorrer declínio do número de lesões por consequência do regime térmico. Nas principais áreas cafeeiras do Paraná, provavelmente serão observados gradientes de infecção em função da altitude (34).

Para as condições do estado de São Paulo, os autores propuseram uma equação que utiliza apenas a temperatura média (Equação 2), conforme Tabela 1 (34). Neste estudo, foi possível observar que o período de incubação diminui com o aumento da temperatura média. Para uma temperatura média de $20^{\circ} \mathrm{C}$ o período de incubação foi de 33 dias (34).

Para efeito comparativo, os autores (34) selecionaram seis municípios, sendo dois do estado do Espírito Santo, dois de Minas Gerias e dois de São Paulo, e estimaram os valores de PI pelas equações 1 (40) e 2 (34). Os resultados dos valores de PI estimados são muito semelhantes (Tabela 3).

Baseando-se na equação 2 (34), também estimaram os valores do PI para 30 municípios do estado do Espírito Santo, entre os meses de outubro a março, considerado como período crítico e mais favorável a evolução da ferrugem do cafeeiro. Os autores observaram que municípios com altitudes inferiores a $400 \mathrm{~m}$, os valores de PI ficaram em torno de 25 dias. Para altitudes de 500 e 600 m, os valores de PI foram de 28 e 29 dias, altitudes de 700 m o PI foi de 30 dias. Já em altitudes de $100 \mathrm{~m}$ o PI foi de 23 dias (34). Corroborando com os resultados encontrados pela a equação 1 (40), onde o efeito da altitude pode influenciar nos valores de PI.

Também com o intuito de estimar o período de incubação da ferrugem do cafeeiro para as condições paulistas, em 1976 (31) estudaram em condições de campo, o período de incubação da ferrugem para três regiões climáticas diferentes do estado de São Paulo: Monte Alegre do Sul, Campinas e Pindorama, e compararam com a equação 1 (40). A partir dos resultados desse estudo, os autores sugerem, então, para trabalhos futuros com período de incubação da ferrugem do cafeeiro, a utilização de uma equação de regressão múltipla, onde, o período de incubação é compreendido pelo número de dias desde a germinação e penetração do patógeno nos tecidos da planta até o aparecimento dos sintomas.

Com esse trabalho, os autores sugeriram seis equações diferentes (equações 3, 4, 5, 6, 7 e 8), considerando o cultivo a pleno sol e sombreado; cultivo pleno sol; e cultivo sombreado, em condições de macroclima e microclima, respectivamente, conforme tabela 1 (31).

Nos meses mais quentes do ano, os autores (31) observaram que, o período de incubação era menor, em torno de 28 dias. Já para os meses mais frios, ocorria exatamente o contrário, ou seja, o período de

Tabela 3. Estimativa do período de incubação da ferrugem do cafeeiro pela equação 1 (40) e 2 (34).

\begin{tabular}{lcccc} 
Municípios & UF & Altitude & Rayner & Ortolani \\
\hline Campinas & SP & 663 & 32 & 31 \\
Ribeirão Preto & SP & 621 & 31 & 31 \\
Conceição do Mato Dentro & MG & 675 & 33 & 32 \\
Poços de Caldas & MG & 1199 & 37 & 37 \\
Conceição do Itapemirim & ES & 35 & 28 & 27 \\
Vitória & ES & 36 & 27 & 26 \\
\hline
\end{tabular}


incubação aumentava para aproximadamente 65 dias, demonstrando que a temperatura mais alta está intimamente relacionada com a ocorrência da ferrugem. A temperatura afeta a germinação dos uredósporos do patógeno (48). A temperatura ótima para ocorrer a germinação e a infecção do patógeno causador da ferrugem do cafeeiro, H. vastatrix, varia entre $22^{\circ} \mathrm{C} \mathrm{e} 24^{\circ} \mathrm{C}$, sendo $30^{\circ} \mathrm{C}$ o limite superior e $15^{\circ} \mathrm{C}$ o inferior $(13,48)$. Entretanto, em condições de campo (10) observou o intervalo de $18^{\circ} \mathrm{C}$ e $26^{\circ} \mathrm{C}$ como ideal para esporulação ou sinais da ferrugem.

Outros autores constataram que temperaturas elevadas interferem na infecção da ferrugem, independente da umidade. Os níveis de infecção da ferrugem ficaram estáveis, em torno de 0,2 pústulas por folha, em condições ótimas de umidade e com altas temperaturas $\left(34,6^{\circ} \mathrm{C}\right)$. Já nos meses mais frios, onde as temperaturas médias oscilam entre $19,8^{\circ} \mathrm{C}$ e $22,2^{\circ} \mathrm{C}$ e apresentam forte seca, o nível de infecção atingiu 2,3 pústulas por folha (31). Os mesmos autores, avaliando o período de incubação do patógeno, em condições microclimáticas a pleno sol, observaram que temperaturas muito elevadas, acima de $31{ }^{\circ} \mathrm{C}$, promoviam um efeito depressivo sobre o período de incubação, quando comparado em condições de sombra, onde as temperaturas são amenas (31).

Estudo realizado para cinco regiões cafeicultoras do estado de São Paulo (Franca, Espírito Santo do Pinhal, Campinas, Vera Cruz e Votuporanga), comparou dados de PI observados em mudas de café inoculadas (16) e PI estimados pela equação 1 (40). Para a região de Franca, os valores de PI observados foram de 40 dias e do PI estimado pela equação foi de 29 dias; de Espírito Santo do Pinhal foi de 38 e 29 dias; de Campinas de 37 a 28 dias; de Vera Cruz de 28 e 31 dias e de Votuporanga de 36 e 25 dias, respectivamente (16).

$\mathrm{Na}$ década de 80 , outros autores (22) estudaram o período de incubação da ferrugem do cafeeiro em Viçosa, estado de Minas Gerais, e desenvolveram equações, utilizando dados de temperatura máxima e mínima. O estudo foi realizado com plantas de café cultivadas em vasos e em condições de sombreamento. Os autores determinaram duas equações de período de incubação: equação (9), foi elaborada em condições de macroclima, em que os dados foram coletados numa estação meteorológica a 100 metros de distância das plantas analisadas e equação (10), foi elaborada com dados de microclima, ou seja, os dados foram coletados exatamente onde estavam as plantas (Tabela 1). Os autores observaram que o período de incubação variou de 29 a 62 dias.

A maioria dos trabalhos realizados no Brasil, para estudar o PI da ferrugem do cafeeiro, utilizam a equação 3 (31). Objetivando desenvolver um modelo de alerta da ferrugem do cafeeiro $(27,28)$ utilizou dados de PI como atributos, num modelo de árvore de decisão.

Neste sentido, outro estudo regionalizado (36) estimou o PI da ferrugem do cafeeiro para o município de Campinas, no período de 1999 e 2014, utilizando a equação 3 (31). Os resultados indicam que o PI variou de 38 a 43 dias. No último quinquênio os autores detectaram uma redução nos valores de PI ao longo do ano, principalmente nos meses de dezembro a fevereiro e junho a agosto.

Com o intuito de simular cenários futuros de epidemias de ferrugem do cafeeiro, em função do PI, com dados de modelos climáticos de projeções futuras, vários estudos $(17,4,18,5,2$ e 4), utilizaram a equação 3 (31).

Considerando dois cenários futuros globais, de alta e baixa emissões de gases de efeito estufa, os autores (17) concluíram que, em ambos os cenários, houve uma tendência de redução de período de incubação, sugerindo um aumento da incidência da doença no futuro.

Posteriormente, em estudo (4) baseado em dados dos modelos climáticos propostos no quarto relatório do Painel Internacional de Mudanças Climáticas - IPCC (AR4) utlizando o modelo climático regional Eta (29), com resolução espacial de $40 \mathrm{~km}$ e alta sensibilidade (23), em cenários de altas emissões de $\mathrm{CO}_{2}$. $\mathrm{O}$ trabalho foi realizado para o período climático "atual", que compreende a normal climatológica de 1961-1990 e, para o cenário projetado“futuro" 2011 a 2040, para os municípios do estado de São Paulo (Franca e Mococa) e Minas Gerais (Machado e São Roque de Minas). Os resultados demonstraram que, no cenário futuro, os valores de PI serão reduzidos durante todo o ano, indicando um maior potencial de infeção da doença. As reduções serão maiores em Franca (30-34 dias para 25-27 dias), no estado de São Paulo e menores em Machado (de 43-44 dias para 41-44 dias), Minas Gerais. $\mathrm{O}$ estudo sugere um aumento da ferrugem do cafeeiro em um futuro climático diferente do atual (4).

Também baseados no quarto relatório do IPCC (AR4), mas utilizando modelos climáticos globais os autores (18) simularam cenários futuros do PI da ferrugem do cafeeiro. Os autores elaboraram mapas de distribuição geográficas da doença utilizando critérios de lógica matemática. Os resultados demostraram que existe uma tendência de aumento da doença ao longo dos anos, corroborando com os resultados encontrados anteriormente $(17,4)$.

Com dados do modelo climático regional Eta-HadGEM2-ES, do $5^{\circ}$ relatório do IPCC (AR5), para os anos de 2020 a 2050, em 2015, e com uma melhor resolução espacial, um novo estudo foi realizado para quatro municípios do estado de Minas Gerais: Guaxupé, Machado, São Lourenço e São Roque de Minas (6). Os resultados também indicaram redução do PI em todos os municípios estudados. Guaxupé reduziu de 38 dias do clima atual para 25 dias no clima futuro; Machado reduziu de 38 dias no clima atual para 30 dias no futuro; São Lourenço reduziu de 38 dias para 36 dias no clima futuro e; São Roque de Minas reduziu de 39 dias no cenário atual para 31 dias no cenário futuro. Esses resultados sugerem que, de maneira geral, considerando-se o modelo Eta-HadGEM2-ES, os municípios deverão apresentar uma maior vulnerabilidade à doença no cenário futuro (6).

Ampliando os estudos com modelos, outros trabalhos $(1,4)$ utilizaram dados de dois modelos climáticos regionais, Eta-HadGEM2ES e Eta-MIROC5, com resolução espacial de $20 \mathrm{~km}$. Para estimar o PI da ferrugem do cafeeiro, foram utilizados dados de temperatura máxima e mínima dos modelos, do período de 1961 a 1990 (normal climatológica), e de 2011 a 2040 (cenário "futuro"), do AR5 do IPCC, RCP 8.5. Para ambos os modelos, no futuro, pode haver um aumento na infestação da doença. O modelo Eta-MIROC5 sugere redução de 3 dias no PI e o Eta-HadGEM2-ES de 8,5 dias. Os autores mencionam ainda que epidemia de ferrugem do cafeeiro no Brasil poderá começar mais cedo e estender seu pico de infecção para os meses de junho a agosto.

\section{CONSIDERAÇÕES FINAIS}

A ferrugem do cafeeiro é uma importante doença para a cultura do café arábica e é estudada no mundo desde 1882. Existem pelo menos dez equações que estimam os valores do Período de Incubação (PI) para estudos da severidade da ferrugem do cafeeiro arábica. Cada uma das equações foi desenvolvida em uma condição específica, e abrange condições de microclima, macroclima, cultivo pleno sol, cultivo sombreado, além de equações gerais, 1 (40) e 2 (34). A maioria dos estudos brasileiros utilizam a equação 3 (31), desenvolvidas para cultivo a pleno sol e sombreado em condição de macroclima, para estudo da severidade da doença, uma vez que essa equação foi desenvolvida para as principais regiões produtoras de São Paulo se adaptando bem às regiões cafeeiras de Minas Gerais. 
Nesse sentido, o PI é um fator importante a ser considerado em estudos de doenças de plantas, em diferentes níveis hierárquicos: local (propriedade), regional e nacional. Estudos são realizados utilizando equações de PI tanto em cenários climáticos atuais quanto em cenários de mudanças climáticas, que implicam em tomadas de decisão e políticas públicas para mitigar problemas futuros e adaptar a cafeicultura nacional, auxiliando agricultores no planejamento e gerenciamento da área.

\section{REFERÊNCIAS}

1. Alfonsi, W.M.V. Vulnerabilidade do cafeeiro arábica à ferrugem (Hemileia vastatrix) em cenários de mudanças climáticas. 2017. 176 p. Tese (Doutorado em Engenharia Agrícola) - Faculdade de Engenharia Agrícola, Universidade Estadual de Campinas, Campinas.

2. Alfonsi, W.M.V.; Koga-Vicente, A.; Pinto, H.S.; Alfonsi, E.L.; Coltri, P.P.; Zullo Junior, J.; Patricio, F.R.A.; Avila, A.M.H.; Goncalves, R.R.V. Climate Change impacts on coffee rust disease. In: AGU Fall Meeting, 49, 2016, San Francisco Anais. San Francisco: AGU, 2016. Disponível em: <http:/ abstractsearch.agu.org/meetings/2016/FM/GC51A-1133.html >. 2016b

3. Alfonsi, R.R.; Ortolani, A.A.; Pinto, H.S.; Pedro Júnior, M.J.; Brunini, O. Associação entre nível de infecção da ferrugem do cafeeiro, variáveis climáticas e área foliar, observados em Coffea arabica L. In: Congresso Brasileiro Sobre Pesquisas Cafeeiras, 2, 1974, Poços de Caldas. Anais. Rio de Janeiro: IBC, 1974. p.80-83.

4. Alfonsi, W.M.V.; Coltri, P.P.; Patricio, R.A.; Zullo Junior, J.; Gonçalves, R.R.V. 2014. Vulnerability of coffee crop to coffee rust disease in Brazil in the high $\mathrm{CO}_{2}$ emission of ETA Regional Climatic Model. In: The 25th International Conference on Coffee Science, 25, 2014, Armenia, Colômbia. Anais. Armenia: ASIC, 2014, p.209 - 209.

5. Alfonsi, W.M.V.; Coltri, P.P.; Zullo Junior, J.; Patricio, F.R.A.; Koga-Vicente, A.; Alfonsi, E.L. Incubation period of coffee rust disease under future climate change scenarios: a case study of Minas Gerais and São Paulo, Brazil. In: XVI Reunión Argentina de Agrometeorología y VIII Reunión Latinoamericana de Agrometeorología, 16, 2016, Puerto Madryn. Anais. RALDA, 2016 a.

6. Alfonsi, W.M.V.; Zullo Junior, J.; Coltri, P.P.; Patricio, F.R.A.; Pereira, V.R.; Gonçalves, R.R.V. Período de Incubação da Ferrugem do Cafeeiro no Cenário de Alta Emissão de $\mathrm{CO}_{2}$ no Estado de Minas Gerais. In: XIX Congresso Brasileiro de Agrometeorologia, 19, 2015, Lavras. Anais. Porto Alegre: SBAgro-Sociedade Brasileira de Agrometeorologia, 2015.

7. Associação Brasileira da Indústria de Café. Estatística. Rio de Janeiro, 2016. Disponível em: $<$ http://www.abic.com.br/publique/cgi/cgilua.exe/sys/start. htm?sid=61\#1910>. Acesso em: 10 fev. 2017.

8. Bergamin Filho, A.; Amorim, L. Doenças com período de incubação variável em função da fenologia do hospedeiro. Fitopatologia Brasileira, Brasília, v.27, p.561-565, 2002.

9. Berkeley; B. Hemileia vastatrix Berk. \& Broome. Gardeners' Chronicle, London, England, v.6, p.1157, 1869.

10. Boldini, J.M. Epidemiologia da ferrugem e da cercosporiose em cafeeiro irrigado e fertirrigado. 2001. 67p. Dissertação (Mestrado em Agronomia) - Universidade Federal de Lavras, Lavras. Disponível em: <http://www. sbicafe.ufv.br/handle/10820/2747>. Acesso em: 10 fev. 2014.

11. Brown, J.S.; Kenny, M.K.; Whan, J.H.; Merriman, P.R. The effect of temperature on the development of epidemics of coffee leaf rust in Papua New Guinea. Crop Protection, v. 14, n.8, p. 671-676, 1995.]

12. Business Insider. Incredible Facts about the Global Coffee Industry. New York, 2014. Disponível em: <http://www.businessinsider.com/facts-aboutthe-coffee-industry-2011-11\#after-crude-oil-coffee-is-the-most-soughtcommodity-in-the-world-1>. Acesso em: 10 fev. 2017.

13. Carvalho, V.L.; Chalfoun, S.M. Doenças do cafeeiro: diagnose e controle. Belo Horizonte: EPAMIG, 2000. 44p. (Boletim Técnico, 58).

14. Carvalho, V.L.; Chalfoun, S.M.; Cunha, R.L. Manejo de doenças do cafeeiro. In: Reis, P.R.; Cunha, R.L. Café arábica: do plantio a colheita. Lavras: EPAMIG, 2010. v.1, p.689-756.

15. Fazuoli, L.C.; Oliveira, A.C.B.; Toma-Braguini, M.; Silvarolla, M.B. Identificação e uso de fontes de resistência durável à ferrugem da folha do café no IAC. In: Zambolim, L.; Maciel-Zambolim, E.; Várzea, V.M.P. (Ed.). Resistência durável à ferrugem da folha do café. Viçosa: UFV, 2005. p.53-74.

16. Figueiredo, P.; Mariotto, P.R.; Silveira, A.P.; Geraldo Junior, C. Período de incubação, evolução de Hemileia vastatrix Berk. \& Br. e utilização da equação de Rayner em alguns municípios cafeeiros do Estado de São Paulo. O Biológico, São Paulo, v.43, p.32-40, 1977.

17. Ghini, R.; Hamada, E.; Pedro Junior, M.J.; Gonçalves, R.R.V. Incubation period of Hemileia vastatrix in coffee plants in Brazil simulated under climate change. Summa Phytopathologica, Botucatu, v.37, n.2, p.8593, abr./jun. 2011. Disponível em: <http://dx.doi.org/10.1590/S010054052011000200001>. Acesso em: 29 abr. 2014.

18. Hamada, E.; Volpato, M.M.L.; Ferreira, G.L.; Alves, H.M.R.; Souza, V.C.O.; Vieira, T.G.C. Simulação dos efeitos das mudanças climáticas sobre a ferrugem do café na região Sudeste do Brasil. In: XVII Simpósio Brasileiro de Sensoriamento Remoto - SBSR, 17, 2015, João Pessoa-PB. Anais. São José dos Campos: INPE, 2015. p. 2629- 2636.

19. IBC - Instituto Brasileiro do Café. Controle da ferrugem do cafeeiro: Verificação da aplicabilidade da equação de Rayner para a determinação do período de incubação de Hemileia vastatrix no Espírito Santo: Subsídios à II Reunião do comitê executivo da comissão nacional de pesquisa com o cafeeiro. Rio de Janeiro: IBC-GERCA, 1973.

20. International Coffee Organization. Belo Horizonte declaration. London, 2013. Disponível em: $<$ http://www.ico.org/documents/cy2012-13/belo-horizonte-declaration-e.pdf $>$. Acesso em: 10 fev. 2017.

21. International Coffee Organization. Total production by all exporting countries. London, 2017. Disponível em: $<$ http://www.ico.org/prices/po-production.pdf>. Acesso em: 15 fev. 2017.

22. Kushalappa, A.C.; Martins, C.P. Incubation periods for Hemileia vastatrix on coffee in Viçosa, Minas Gerais. Fitopatologia Brasileira, Brasília, DF, v.6, n.1, p.177-183, 1980.

23. Marengo, J.A.; Chou, S.C.; Kay, G.; Alves, L.M.; Pesquero, J.F.; Soares, W.R.; Tavares, P. Development of regional future climate change scenarios in South America using the Eta CPTEC/HadCM3 climate change projections: climatology and regional analyses for the Amazon, São Francisco and the Paraná River basins. Climate Dynamics, Cham, v.38, n.9/10, p.1829-1848, 2012.

24. Matiello. J.B.; Almeida, S.R. A ferrugem do cafeeiro no Brasil e seu controle. Varginha: Bom Pastor, 2006. 106p.

25. Mayne, W.W. Seasonal periodicity of coffee leaf disease (Hemileia vastatrix), B. Br.). Bulletin Mysore Coffee Experiment Station, Bangalore, n.4, p.22, 1930.

26. Medeiros, A.G. Informe sobre Hemileia vastatrix em café na Bahia, Brasil Rio de Janeiro: CEPLAC, 1970.

27. Meira, C.A.A. Processo de descoberta de conhecimento em bases de dados para a análise e o alerta de doenças de culturas agrícolas e sua aplicação na ferrugem do cafeeiro. 2008. 478p. Tese (Doutorado em Engenharia Agrícola) - Faculdade de Engenharia Agrícola, Universidade Estadual de Campinas, Campinas. Disponível em: $<$ http://www.bibliotecadigital.unicamp.br/document/?code=vtls000443483\&fd=y>. Acesso em: 30 abr. 2013.

28. Meira, C.A.A.; Rodrigues, L.H.A.; Moraes, S.A. Modelos de alerta para o controle da ferrugem do cafeeiro em lavouras com alta carga pendente. Pesquisa Agropecuária Brasileira, Brasília, DF, v.44, n.3, p.233-242, mar. 2009.

29. Mesinger, F.; Chou, S.C.; Gomes, J.L.; Jovic, D.; Bastos, P.; Bustamante, J.F.; Laziac, L.; Lyra, A.A.; Morelli, S.; Ristic, I.; Veljovic, K. An Upgraded Version of the Eta Model. Meteorology and Atmospheric Physics, Roskilde, v.116, p.63-79, 2012.

30. Monaco, L.C.; Scali, M.H.; Fazuoli, L.C.; Sondahl, M. R. Variabilidade na área foliar do cafeeiro. In: XXIV Reunião Anual da SBPC, 24, 1972, São Paulo. Anais. São Paulo: Ciência e Cultura, 1972. 402 p.

31. Moraes, S.A.; Sugimori, M.H.; Ribeiro, I.J.A.; Ortolani, A.A.; Pedro Jr., M.J. Período de incubação de Hemileia vastatrix Berk. et Br. em três regiões do Estado de São Paulo. Summa Phytopathologica, Piracicaba, v.2, n.1, p.32-38, 1976.

32. Moraes, S.A. A ferrugem do cafeeiro: importância, condições predisponentes, evolução e situação no Brasil. Campinas: Instituto Agronômico, 1983. $50 \mathrm{p}$.

33. Nutman, E.J.; Roberts, F.M. Studies on the biology of Hemileia vastatrix Berk. et Br. Transactions of Britisth Mycological Society, Cambridge, v.46, n.1, p.27-48, Jan. 1963. 
34. Ortolani, A.A.; Viana, A.C.C.; Abreu, R.G. Hemileia vastatrix Berk et br. estudos e observações em regiões da África e sugestões a cafeicultura do Brasil. Rio de Janeiro: IBC-GERCA, 1971. 193p. Relatório de missão realizada em Angola, África do Sul, Quênia, Tanzânia, Uganda e instituições de pesquisa em relação à ferrugem do cafeeiro.

35. Ortolani, A.A. Contribuição ao estudo ecológico da ferrugem do cafeeiro em diferentes populações de Coffea arabica $\mathrm{L}$. na região de Pindorama. 1973. 91p. Tese (Doutorado em Agrometeorologia) - Universidade Estadual Paulista, Botucatu.

36. Pantano, A.P.; Patrício, F.R.A.; Alfonsi, W.M.V.; Meireles, E.J.L. Condições climáticas e período de incubação para ferrugem do cafeeiro nos anos de 2013 e 2014 na região de Campinas, SP. In: IX Simpósio de Pesquisa dos Cafés do Brasil, 9, 2015, Curitiba. Anais. Brasília: Consórcio Brasileiro de Pesquisa e Desenvolvimento do Café, 2015. Disponível em: $<$ https://ainfo. cnptia.embrapa.br/digital/bitstream/item/140606/1/Condicoes-climaticas. pdf>. Acesso Mar. 2017.

37. Patrício, F.R.A.; Oliveira, E.G. Desafios do manejo no controle de doenças do café. Visão Agrícola, Piracicaba, v. 12, p.51-54, 2013.

38. Paul, V. Biologia y propagación de Hemileia vastatrix Berk \& Br., patógeno da La roya del café. Pflanzenschutz - Nachrichten Bayer, Leverkusen, v.33, n.2, p.97-107, 1980.

39. Pereira, A.R.; Camargo, A.P.; Camargo, M.P. Agrometeorologia de cafezais no Brasil. Campinas: Instituto Agronômico, 2008. 127p.

40. Rayner, R.W. Germination and penetration studies on coffee rust (Hemileia vastatrix B. \& Br.). Annals of Applied Biology, Wellesbourne, v.49, p.497-505, 1961.
41. Romeiro, R.S. Germinação e poder infectivo dos uredósporos de Hemileia vastatrix Berk. et Br. mantidos sobre diferentes produtos vegetais e o suscetível. 1971. 41p. Dissertação (Mestrado em Microbiologia Agrícola) - Universidade Federal de Viçosa, 1971.

42. Vanderplank, J.E. Plant diseases: epidemics and control. New York: Academic, 1963.

43. Wallis, J.A.N. Coffee leaf rust in South America: a report to the Internacional Coffee Organization. Londres: ICO, 1970. 49p.

44. Ward, H.M. Researches on the Life-history of Hemileia vastatrix, the Fungus of the "Coffee-leaf Disease". Journal of the Linnean Society of London, London, v.19, p.299-335, 1882. doi:10.1111/j.1095-8339.1882.tb00377.x

45. Zambolim, L. Current status and management of coffee leaf rust in Brazil. Tropical Plant Pathology, Brasília, v.41, n.1, p.1-8, 2016. doi: doi. org/10.1007/s40858-016-0065-9

46. Zambolim, L.; Vale, F.X.R.; Pereira, A.A.; Chaves, G.M. Café (Coffea arabica L.): controle de doenças - doenças causadas por fungos, bactérias e vírus. In: Vale, F.X.R.; Zambolim, L. (Ed.). Controle de doenças de plantas: grandes culturas. Viçosa: UFV, 1997. v.1, p.83-139.

47. Zambolim, L.; Vale, F.X.R.; Pereira, A.A.; Chaves, G.M. Manejo integrado das doenças do cafeeiro. In: Zambolim, L. Produção de café com qualidade. Viçosa: Universidade Federal de Viçosa, 1999. p.134-251.

48. Zambolim, L.; Vale, F.X.R., Zambolim, E.M. Doenças do cafeeiro. In: Kimati, H.; Amorim, L.; Rezende, J.A.M.; Bergamin Filho, A.; Camargo, L.E.A. Manual de fitopatologia. 4.ed. São Paulo: Editora Agronômica Ceres, 2005. v.1, p.165-180.

49. Zambolim, L.; Maciel-Zambolim, E.; Vale, F.X.R.; Pereira, A.A.; Sakyiama, N.S.; Caixeta, E.T. Raças fisiológicas de Hemileia vastatrix no Brasil: variabilidade fisiológica, situação atual e perspectivas futuras. In: Zambolim, L.; Maciel-Zambolim, E.; Várzea, V.M.P. (Ed.). Resistência durável à ferrugem da folha do café. Viçosa: UFV, 2005. p.53-74. 